\title{
Rate of force development and the lateralized readiness potential
}

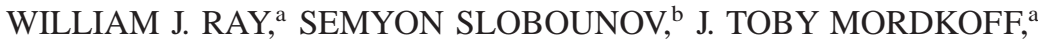 \\ J. JOHNSTON, ${ }^{\mathrm{b}}$ AND ROBERT F. SIMON ${ }^{\mathrm{b}}$ \\ ${ }^{a}$ Department of Psychology, The Pennsylvania State University, University Park, USA \\ ${ }^{b}$ Department of Kinesiology, The Pennsylvania State University, University Park, USA
}

\begin{abstract}
We examined the relationship between force and rate of force development aspects of movement dynamics and electroencephalogram motor components as reflected in the lateralized readiness potential (LRP). Using self-paced tasks, in Studies 1 and 3 we investigated whether differential speed and accuracy constraints in discrete and repetitive finger force production tasks influenced the LRP. These studies showed that speed tasks produced larger LRP than accuracy tasks regardless of whether the movement type was discrete or repetitive. In Studies 2 and 4 we studied four conditions with two levels of force and two levels of rate of force development. The largest LRPs were found with the greatest rate of force development. Overall, the four studies demonstrated that preparation for differential rates of force development is a major component reflected in the LRP.
\end{abstract}

Descriptors: LRP, Rate of force development, Speed and accuracy tasks

Numerous behavioral studies have explored sensorimotor processes involved in preparation for motor acts (e.g., Crossman \& Goodeve, 1983; Fitts, 1954; Hancock \& Newell, 1985; Meyer, Smith, Kornblum, Abrams, \& Wright, 1990). One determining characteristic of these preparatory processes is that of rate of force development. Behaviorally, the rate of force development has been shown to account for systematic changes in reaction time, whereas both peak force and force duration play secondary roles (Carlton, Carlton, \& Newell, 1987). Because little if any electrocortical research has been carried out to examine rates of force development, we conducted two initial studies. In the first study (Slobounov $\&$ Ray, 1998), we investigated whether different speed and accuracy constraints in discrete and repetitive index finger force production movements influence components of movement-related potentials (MRPs) preceding and accompanying these movements. Using three components of MRPs (Bereitschaftspotential, BP; motor potential, MP; and movement-monitoring potential, MMP), we found MMP, but not BP and MP, was enhanced at higher rates of force development both for speed and accuracy tasks. Specifically, a high positive correlation was found between MMP's peak amplitude and the rate of force development for both repetitive and discrete movements. A primary means by which the speed of a

Studies 1 and 2 were presented at the 39th Annual Meeting of the Society for Psychophysiological Research, Denver, September 1998.

This work was supported in part by a grant from NASA (Langley).

We thank Manny Donchin, Cathleen Moore, and Steve Hackley for valuable suggestions concerning this research.

Address reprint requests to: William J. Ray, Department of Psychology, The Pennsylvania State University, University Park, PA 16802, USA. E-mail: wjr@psu.edu. movement can be controlled is by either increasing or decreasing the rate of force development in a particular movement, which may be an explanation for the high correlation. We extended this finding in the second study (Slobounov, Ray, \& Simon, 1998) by a direct examination of the relationship between force and rate of force development and the amplitude of movement related potentials. In that study we found that the amplitudes of movementrelated potential components preceding and accompanying finger force production movements were significantly correlated with rate of force development rather than with force itself. We now extend our previous research by examining how rate of force development influences the lateralized readiness potential (LRP).

The LRP has been described as a measure of response activation and as such is an important tool in the study of the neural basis of human information processing (Hackley \& Miller, 1995). It is based on the BP or readiness potential (RP) first described by Kornhuber and Deecke (1965) that appears at 800-500 ms before a voluntary, self-initiated motor response and reaches its maximum at approximately the time of movement initiation. The RP is maximal at central sites and contralateral to the responding hand (Vaughan, Costa, \& Ritter, 1968). As described elsewhere (e.g., Coles, 1989; Osman, Moore, \& Ulrich, 1995), the LRP is computed by subtracting the ipsilateral potential from the contralateral potential for each hand, which results in a measure reflecting only movement-related parameters. As such the LRP is seen as a measure of motor preparation. Neuroanatomical evidence from surface and depth electrodes suggests that the LRP is generated mainly by the primary motor cortex (see Miller \& Hackley, 1992, for a review).

Various factors have been shown to influence the LRP. For example, Hackley and Miller (1995) reported that larger LRPs 
preceded complex movements (i.e., subjects were requested to press a sequence of three keys, using the index, ring, and middle fingers) as compared with simple movements (i.e., single index finger keystroke was requested). Gratton et al. (1990) showed that valid precues induce contralateral LRPs whereas invalid precues induce ipsilateral LRPs. However, Miller, Coles, and Chakraborty (1996) reported that in response to probe stimuli in a go/nogo situation, priming influenced the reaction time to separate probe stimuli but not the LRP. Further, De Jong, Coles, Logan, and Gratton (1990) studied response inhibition using the LRP. They asked if LRPs could reflect a point of no return in responding and concluded there was no such point and that responses can be inhibited at any time.

Eimer (1998), in his review of the literature, found that LRP influences in conditions defined by velocity and force have not been investigated systematically. In an earlier discussion of BPs and LRPs, Sommer, Leuthold, and Ulrich (1994) suggested that LRP reflects a type of motor preparation independent of force and direction. They tested this assumption by instructing individuals to press a force key in terms of two levels of peak force and two times to peak force. Sommer et al. reported that neither force levels nor rate of force production affected the LRP. Examining the role of advanced information including force level, direction of movement, and response hand on the LRP, Ulrich, Leuthold, and Sommer (1998) reported LRP amplitude increased only for the precue that included information about all three response parameters. Partial information concerning movement direction and response force did not influence the LRP unless both were specified. Ulrich et al. Concluded that their results reflect a strong version of a hierarchical preparation hypothesis in which both response force and movement direction must be available before the next level in the hierarchy can be activated. How rate of force development and its parameters influence the LRP is still unknown. Understanding the relationship between rate of force development and the LRP will allow for better articulation of the level of abstraction of the motor representation that underlies the LRP.

In a reanalysis of previously published data (Slobounov \& Ray, 1998; Slobounov et al., 1998) and a replication and extension of this work with new research, we examined the relationship between rate of force development and the LRP. In Study 1, we examined the LRP in relation to the role of speed and accuracy constraints in discrete and repetitive index finger force production movements. The rate of force development, however, was revealed as a behavioral variable that correlated highly with changes in the LRP. Therefore, in Study 2, we extended this work by a direct examination of the relationship between force and rate of force development and the LRP. To rule out alternative explanations concerning factors that influence the LRP, Studies 1 and 2 were extended in Studies 3 and 4 using a different response hand sequence.

\section{Method}

\section{Subjects}

Subjects in Studies 1, 2, 3, and 4 were college students who were right handed, according to the Edinburgh inventory (Oldfield, 1971) (Study 1: $n=12$, mean age $=22$ years; Study 2: $n=12$, mean age $=23$ years; Study 3: $n=5$, mean age $=21$ years; Study 4: $n=$ 5 , mean age $=22$ years). The participants had no history of pathologies to either the hand or wrist. Informed consent was obtained before the experiment and extra class credit was received for participation.

\section{Experimental Tasks}

Studies 1, 2, 3, and 4. Subjects were seated in an electrically shielded room with the light dimmed for the entire experiment. The subject's arm was placed in a comfortable position on a table with the index finger on a load cell. During the experiment, subjects carried out the required tasks with the right and left hands by pressing the index finger against the load cell. Feedback of the force output was provided via a computer monitor directly in front of the subject. The maximum voluntary force (MVF) for a given subject was determined by asking subjects to press the load cell with their right index finger and then with their left index finger as strongly as possible. The mean values of maximum force production over two trials per subject were computed and defined as MVF.

Studies 1 and 3. Depending on the task, two or three target lines were presented on the screen: the first was a straight horizontal line indicating $50 \%$ of the maximum force the subject could produce with the finger; the second line was a similar line indicating $10 \%$ of the maximum force. A third line indicated directly the force with which the subject pressed on the load cell. This third line was the subject's force trajectory and could be viewed by the subject on the computer screen.

Twenty-five trials of four different tasks were performed by each subject with each hand (100 trials for each hand). Task 1 required the subject to apply pressure on a load cell as accurately as possible until the pressure line on the monitor was equal to the $50 \%$ MVF line and to hold it for $5 \mathrm{~s}$. Task 2 consisted of the subject applying pressure as fast as possible (without an accuracy requirement) to the load cell to reach the 50\% MVF line and to hold it for $5 \mathrm{~s}$. Task 3 required the subject to vary finger pressure on a load cell between the $50 \%$ and $10 \%$ lines (i.e., to reach the upper and lower target lines) as accurately as possible in a 5-s time period. This task produced sine-wave-like curves. Task 4 required the subject to vary finger pressure on a load cell producing the sinewave-like curves within the $50 \%$ and $10 \%$ lines as fast as possible in a 5-s time period. Using these four tasks, both discrete and repetitive isometric force output patterns were examined under conditions of speed and accuracy. The order of presentation of the four tasks was determined by a random number generator. All trials for a given task were completed before progressing to the next. In Study 1, all four tasks were completed for the right hand and then the left. In Study 3 the hands were alternated every fifth trial for each task.

Studies 2 and 4. Subjects were presented with two horizontal blue lines on the screen of the computer monitor. The lower line, approximately 2.5 inches from the bottom of the screen, represented $35 \%$ of the subject's MVF. The upper line, approximately 2 inches above the $35 \%$ line, represented $65 \%$ of the MVF. A yellow line gave subjects feedback in relation to their finger pressure on the load cell. At the beginning of the experiment, subjects were allowed to familiarize themselves with this feedback.

The experimental session consisted of four separate tasks (50 trials of each task in Study 2 and 25 trials in Study 4) in which two levels of force (35\% and 65\% of MVF) and two levels of rate of force development (14.4\% $\mathrm{MVF} / \mathrm{s}$ and $31.8 \% \mathrm{MVF} / \mathrm{s})$ were manipulated experimentally. As in Studies 1 and 3, the tasks were performed in a blocked random order for each index finger $(200$ trials for each hand in Study 2 and 100 trials for each hand in Study 4). In Study 2, the four tasks were performed by the right hand and then by the left. In Study 4, the hands were alternated 
every fifth trial for each task. The rate of force development was computed using peak force of individual trials over time-to-peak force (Pf/Tp) (Newell \& Carlton, 1985). Depending on the task, a dot was shown on either of the two blue lines, in one of two locations. The four locations were as follows: (1) the 1.125-s mark on the $35 \%$ of MVF line, corresponding to $31.8 \% \mathrm{MVF} / \mathrm{s}$; (2) the 2.042 -s mark on the $65 \%$ line, corresponding to $31.8 \% \mathrm{MVF} / \mathrm{s}$; (3) the 2.43-s mark on the $35 \%$ line, corresponding to $14.4 \% \mathrm{MVF} / \mathrm{s}$; and (4) the 4.5 -s mark on the $65 \%$ line, corresponding to $14.4 \%$ $\mathrm{MVF} / \mathrm{s}$. For each task, the subject was instructed to put pressure on the load cell with the index finger in such a way that the slope of the yellow signal would be constant, and to reach the dot presented on the screen. From the point of the force initiation to the point of the dot on the monitor, subjects were instructed to keep the yellow line as straight as possible.

\section{Movement Recording Apparatus}

Force pressure was measured with EL load cells (Entran Devices, Inc.), which register the displacement via a strain gauge bridge incorporated in the cell and transduced via a Coulbourn Instrument TM Transducer Coupler Type A (strain gauge bridge). The electroencephalogram (EEG) signal was converted via a Data Translation TM DT2801-A 12 bit A/D board with a 200-Hz sampling rate. Online feedback was provided to the subject on a $640 \times 480$ VGA monitor.

\section{EEG Recording}

A programmable DC coupled broadband SynAmps amplifier (NeuroScan, Inc., El Paso, TX) with an Electro-Cap electrode (Ag/ $\mathrm{AgCl})$ helmet was used to record EEG at 9 sites (Fz, F3, F4, Cz, $\mathrm{C} 3, \mathrm{C} 4, \mathrm{Pz}, \mathrm{P} 3$, and P4) according to the International 10-20 system (Jasper, 1958) referred to linked ears. Electrode impedances were below $5 \mathrm{k} \Omega$. $\mathrm{Ag} / \mathrm{AgCl}$ electrodes were placed supra- and suborbitally to the right eye and $2 \mathrm{~cm}$ external to the outer canthus of each eye to record the horizontal and vertical movements in the electrooculogram (EOG). Because subjects were instructed to focus on the computer screen, there were limited eye movements. The EEG signals were amplified (gain 2,500, accuracy 0.033/bit) with a recording range set for $\pm 55 \mathrm{mV}$ in the $\mathrm{DC}$ to $70-\mathrm{Hz}$ frequency range. The EEG data were digitized at $200 \mathrm{~Hz}$ using 16-bit analog-to-digital converters. The recording epoch $(2,000 \mathrm{~ms}$ preceding and $1,000 \mathrm{~ms}$ following the peak of finger force output) was triggered by the signal from the load cell when force level crossed the criterion of 5\% MVF. Electrode DC shift was compensated for offline by a fourth order trend correction of each channel over the entire recording epoch to remove a drift in the data that extends beyond the sample epoch (linear detrend option of NeuroScan software). The baseline was derived from the average of the segment from 2,000 to $1,800 \mathrm{~ms}$ before the trigger point for each channel. Digitized single-trial EEG and EOG data synchronized with force production records were processed by the NeuroScan 3.1 software package.

\section{Data Reduction and Analysis}

Studies 1 and 3. The characteristics of the force-time impulses were examined separately for discrete and repetitive tasks. The accuracy of finger force output during discrete tasks (i.e., maintenance phase) was estimated by computing the root mean square (RMS) representing the amount of force error relative to the required force criterion. To estimate the time to target, the final $3 \mathrm{~s}$ of the trial duration was used. A median point of overshoot from the first crossing point of the target line was calculated. In addition, rate of force development was computed to examine the initial phase of the force output. The error of this initial rate of force phase was computed based on deviation of peak force level from the required force (i.e., RMS).

The level of performance on the repetitive force production tasks was estimated by computing the RMS, SD (standard deviation), and $\mathrm{CoV}$ (coefficient of variation) of the load cell data with respect to the $50 \%$ and $10 \%$ of MVF target lines and averaged force values both for peaks and troughs. The speed of repetitive finger tasks was estimated by computing the number of peaks and troughs over the entire trial duration and the number of output cycles per second. In addition, the initial phase of the repetitive finger force production task was estimated by computing the rate of force development in the first cycle and the deviation of peak force level from the required force. Representative examples of subjects' performance on the discrete speed and accuracy finger force-production tasks are shown in Figure 1.

Studies 2 and 4. Force production data were examined separately for each task as described previously. The two force values (peak force and the rate of force development) were averaged over 50 trials for each task in Study 2 and 25 trials in Study 4. Preliminary analyses of raw data of force production tasks showed considerable variability between subjects' MVF within 6-12 N. Therefore, standard normalization procedures were used. In particular, data from individual trials were divided by MVF data for a given subject and multiplied by 100 . The analysis of the accuracy of force production data was produced based on the normalized data set. A representative example of a subject's performance on the four required tasks is shown in Figure 2.

EEG and LRP. EEG continuous data sets were epoched and averaged. Fifty sweeps were averaged following artifact correction. Studies 1 and 2 were corrected in terms of vertical eye movement. Studies 3 and 4 were corrected in terms of both vertical and horizontal eye movements. In particular, the transmission of EOG into the EEG was estimated by linear regression in areas of maximum EOG variance. EEG was then corrected for blinks by subtracting the blinks as measured in the EOG weighted by a transmission coefficient. An average EEG for each task (25 trials per task in Studies 1, 3, and 4 and 50 trials per task in Study 2) for each subject under the task conditions was calculated time-locked to the onset of force production, and the grand average for the subjects was calculated. The LRPs were calculated according to De Jong, Wierda, Mulder, and Mulder (1988). Specifically, the LRP was derived from the following equation: left finger (C3$\mathrm{C} 4)$ - right finger (C3-C4), where left and right refer to the hand designated by the experimental design (self-paced finger movement) and $\mathrm{C} 3-\mathrm{C} 4$ is the difference between the two electrode sites. Following this procedure, the resulting LRP will be positive when there is more negativity contralateral to the response hand, negative when there is greater electrical potential ipsilateral to the response hand, and zero when the potential is unaffected by the identity of the signaled hand (Osman \& Moore, 1993). As described in Hackley and Miller (1995), LRPs used in the analysis were from the 400-ms period preceding response.

In Studies 1 and 3, a $2 \times 2$ repeated-measures analysis of variance (ANOVA) using task requirement (speeded [Spd] and accurate $[\mathrm{Acc}]$ ) and movement types (discrete [Dis] and repetitive [Rep]) was used to analyze the effect of the experimental manip- 

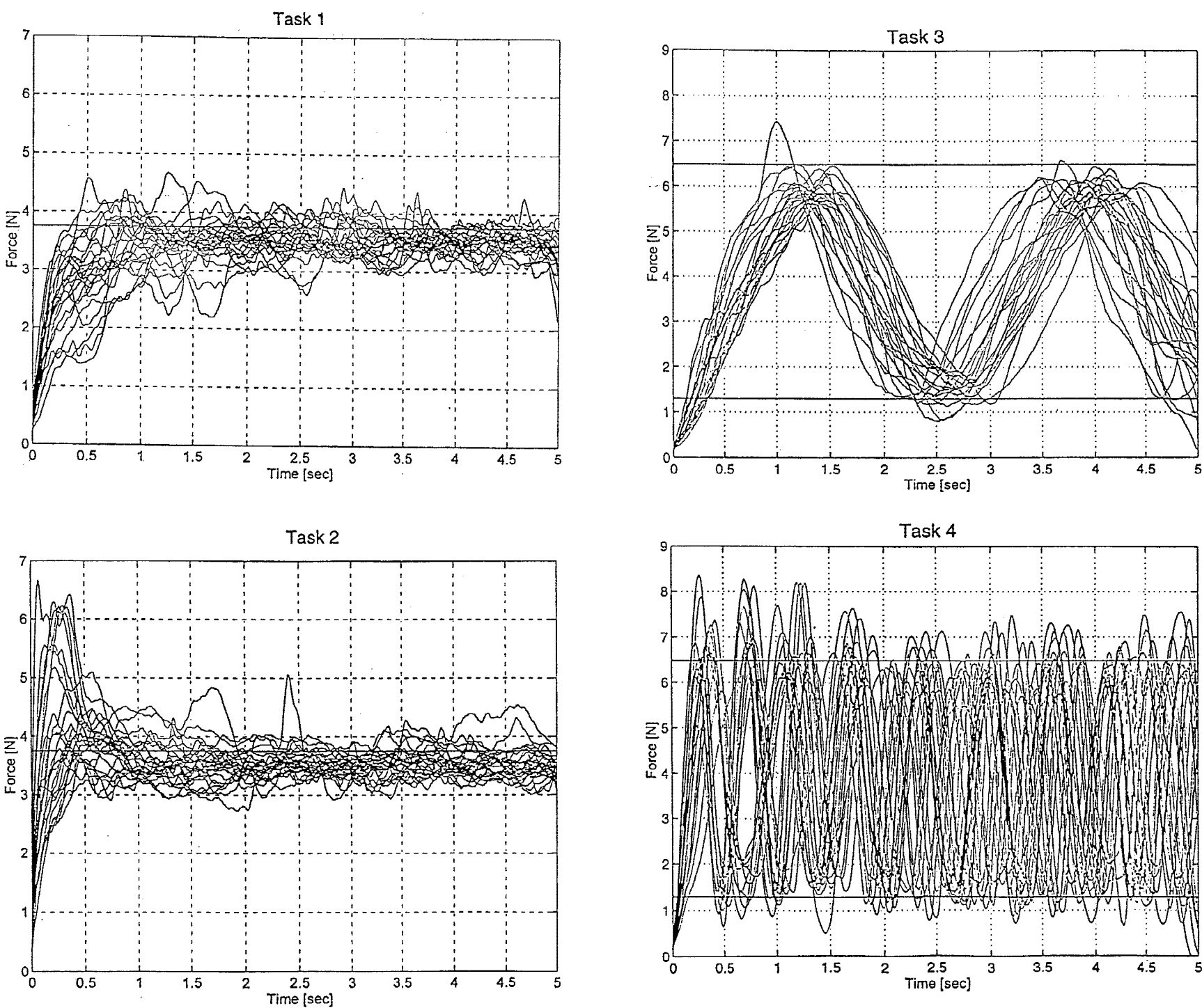

Figure 1. Representative examples of discrete and repetitive finger force production tasks from a single subject. Task 1 is a discrete accuracy task; Task 2 is a discrete speed task; Task 3 is a repetitive accuracy task. Task 4 is a repetitive speed task.

ulations on the LRP. In Studies 2 and 4, a $2 \times 2$ repeated-measures ANOVA using the two levels of force (lower force [Lf] and higher force [Hf]) and the two levels of rate of force development (lower rate of force development [Lrfd] and higher rate of force development [Hrfd]) was used to analyze the effect of the experimental manipulations on the LRP.

\section{Results}

\section{Behavioral Data}

Studies 1 and 3. In Study 1 (as reported in Slobounov \& Ray, 1998), for the discrete tasks, there were significant differences in the force error (RMS) for speed and accuracy instructions, $F(1,11)=$ $8.15, p<.01$. The mean peak force was higher for the speed instruction and significantly different from the accuracy instruction, $F(1,11)=3.49, p<.01$. Accordingly, the rate of force development was significantly higher for the speed instruction, $F(1,11)=12.85, p<.01$. The main effect of side initiating the force output (i.e., right vs. left finger force production within a task) was not significant. In terms of repetitive tasks, there were significant differences in the force error of the first cycle (RMS) between speed and accuracy tasks, $F(1,11)=5.85, p<.01$. The mean peak force for this first cycle was higher for the speed task and significantly different from the accuracy task, $F(1,11)=4.18$, $p<.01$. The rate of force development was significantly higher for the speed instruction, $F(1,11)=5.30, p<.01$. Similar to the discrete task, the main effect of force-production side (i.e., right vs. left finger force production within a task) was not significant.

In Study 3, for the discrete tasks, there were significant differences in the force error (RMS) for speed and accuracy instructions, $F(1,9)=6.98, p<.05$. The mean peak force was higher for the speed instruction and significantly different from the accuracy instruction, $F(1,9)=2.42, p<.05$. Accordingly, the rate of force development was significantly higher for the speed instruction, $F(1,9)=7.37, p<.01$. Again, the main effect of side initiating the force output (i.e., right vs. left finger force production within 

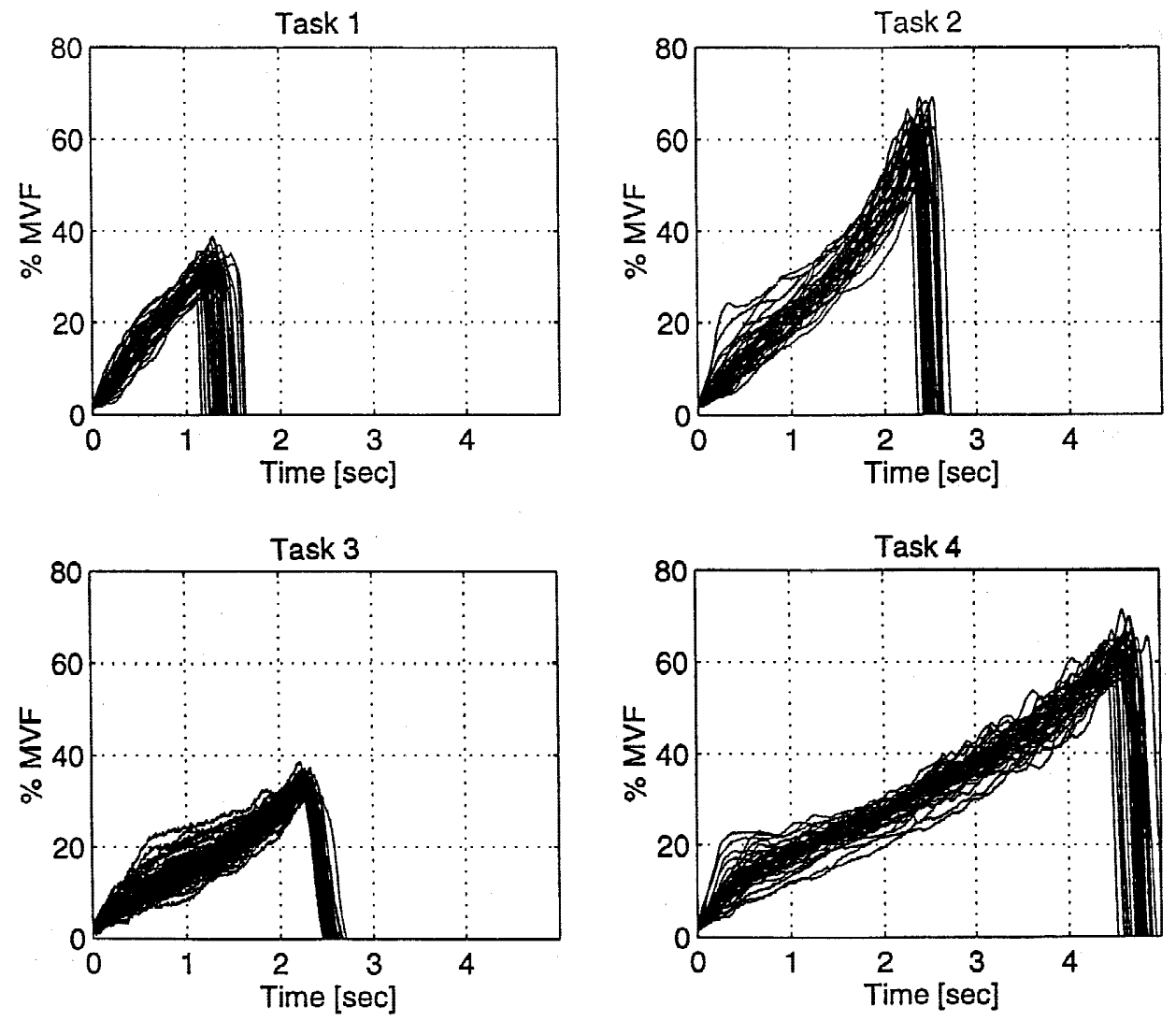

Figure 2. Representative examples of four finger force production tasks from a single subject. Task 1 requires lower level of force and higher rate of force development. Task 2 requires higher level of force and higher rate of force development. Task 3 requires higher level of force and lower rate of force development. Task 4 requires lower level of force and lower rate of force development. (MVF $=$ maximum voluntary force.)

a task) was not significant. In terms of repetitive tasks, there were significant differences in the force error of the first cycle (RMS) between speed and accuracy tasks, $F(1,9)=4.53, p<.05$. The mean peak force for this first cycle was higher for the speed task and significantly different from the accuracy task, $F(1,9)=5.24$, $p<.01$. The rate of force development was significantly higher for the speed instruction, $F(1,9)=4.53, p<.05$. Similar to the discrete task, the main effect of force-production side (i.e., right vs. left finger force production within a task) was not significant.

Studies 2 and 4. For Study 2 (as reported in Slobounov et al., 1998), no significant differences were observed in accuracy of force production in the time domain, $p>.05$. Further, RMS as an indication of force production error was significantly higher for the 65\% MVF tasks (i.e., Tasks 2 [HfHrfd] and 4 [HfLrfd]) than for the $35 \%$ MVF tasks (Tasks 1 [LfHrfd] and 3 [LfLrfd]), $F(1,11)=$ $9.41, p<.05$. The correlation between force and the RMS of force production was 0.52 and that between time and RMS for time was 0.33 . There was a significant main effect for rate of force development as a function of task, $F(3,33)=528.33, p<.001$. Post hoc analyses revealed that the rate of force development was higher for Tasks 1 (DisAcc) and 2 (DisSpd) than for Tasks 3 (RepAcc) and 4 (RepSpd) $(p<.001)$. Moreover, there was a significant main effect of rate of force development error (i.e., RMS), $F(3,33)=$ $37.43, p<.01$, which suggests that subjects were less accurate in tasks with higher rates of force production. A high positive correlation also existed between the rate of force development and its error $(r=.74)$.

For Study 4, analyses of behavioral data revealed trends similar to Study 2. First, overall the main effect of force-production side (i.e., right vs. left finger force production within a task) was not significant regardless of task. Therefore, in the following analyses the behavioral data from left and right sides were combined to assess main effects of force and rate of force development. No significant differences were observed in accuracy of force production in the time domain, $p>.05$. Further, RMS of force production was significantly higher for the $65 \%$ MVF tasks (i.e., Tasks 2 [HfHrfd] and 4 [HfLrfd]) than for the 35\% MVF tasks (Tasks 1 $[\mathrm{LfHrfd}]$ and 3 [LfLrfd]), $F(1,36)=3.42, p<.05$. The correlation between force and the RMS of force production was 0.42 and that between time and RMS for time was 0.63 . There was a significant main effect for rate of force development as a function of task, $F(1,36)=68.45, p<.01$. As expected the rate of force development was higher for Tasks 1 (LfHrfd) and 2 (HfHrfd) than for Tasks 3 (LfLrfd) and 4 (HfLrfd) $(p<.01)$. Moreover, there was a significant main effect of rate of force development error (i.e., RMS), $F(1,36)=11.32, p<.01$, which suggests that subjects were less accurate in tasks with higher slopes of force production. A high positive correlation also existed between the rate of force development and its error $(r=.78)$. 


\section{Electrophysiological Data}

Study 1. Grand-average waveforms of the force-onset synchronized LRP for the four experimental tasks are shown in Figure 3. As noted all LRPs were calculated using a window beginning $400 \mathrm{~ms}$ prior to the response and ending at the response. As can be seen from this figure, the largest LRP $(1.399 \pm 0.497 \mu \mathrm{V})$ was observed when subjects performed the repetitive task as fast as possible (RepSpd task). Less, but still significant lateralization $(0.755 \pm 0.139 \mu \mathrm{V})$ was observed for the discrete task under the speed condition (DisSpd task). Nonsignificant lateralization was observed when subjects performed the repetitive task under the accuracy conditions (RepAcc task) $(0.345 \pm 0.194 \mu \mathrm{V})$. Negative lateralization was observed during the discrete task under accuracy conditions (DisAcc task) $(-0.298 \pm 0.254 \mu \mathrm{V})$. In particular, the ANOVA revealed significant differences between speed and accuracy tasks, with larger LRP for speed tasks, $F(1,11)=6.93, p<$ .01 . There also was a significant effect for movement type with the overall tendency toward larger LRP for repetitive movement, $F(1,11)=5.50, p<.05$. There was a significant interaction of movement type (discrete vs. repetitive) and instruction (speed vs. accuracy), $F(1,11)=4.61, p<.05$. A Newman-Keuls post hoc analysis revealed that LRP values for RepSpd task were significantly larger than those for RepAcc task $(p<.01)$. LRP values for DisSpd task were significantly higher than those for DisAcc task $(p<.01)$. In addition, LRP values for RepSpd task were significantly higher than those for DisSpd task $(p<.05)$.

Study 2. Grand-average waveforms of the force-onset synchronized LRP for the four experimental tasks are shown in Figure 4. As can be seen from this figure, the largest $\operatorname{LRP}(2.868 \pm 0.338 \mu \mathrm{V})$ was observed during Task 2 (HfHrfd), which required the highest nominal force $(65 \% \mathrm{MVF})$ and a higher rate of force development (31.8\% MVF/s). Slightly lower LRP values $(2.561 \pm 0.248 \mu \mathrm{V})$ were observed when the same rate of force development $(31.8 \%$ $\mathrm{MVF} / \mathrm{s}$ ) with less nominal force level (35\% MVF) was required (Task 1, LfHrfd). Even lower, but still significant LRP values $(1.262 \pm 0.535 \mu \mathrm{V})$ were observed when subjects performed Task 3

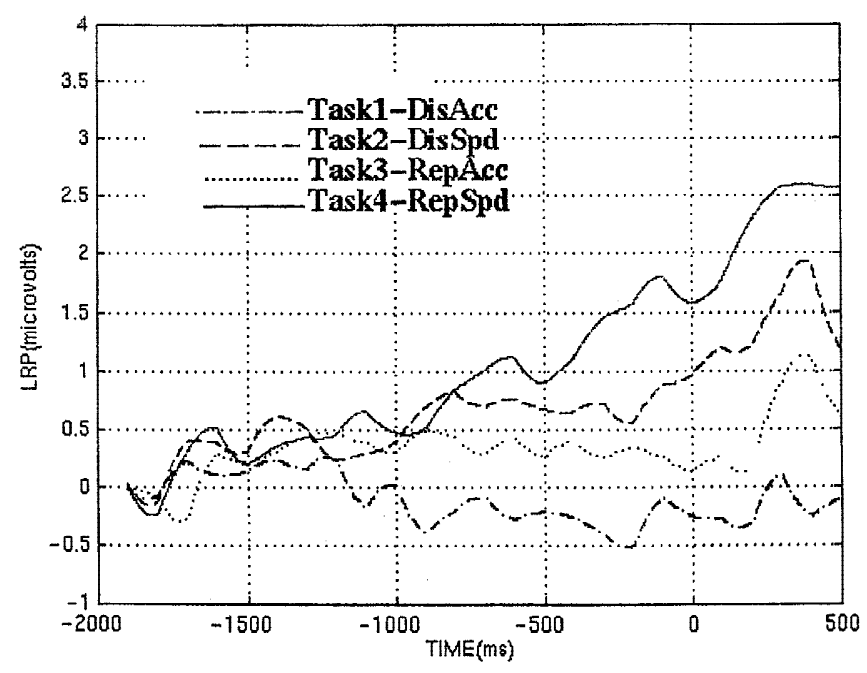

Figure 3. Lateralized readiness potentials from Study 1. Task 1 is a discrete accuracy task; Task 2 is a discrete speed task; Task 3 is a repetitive accuracy task; Task 4 is a repetitive speed task.

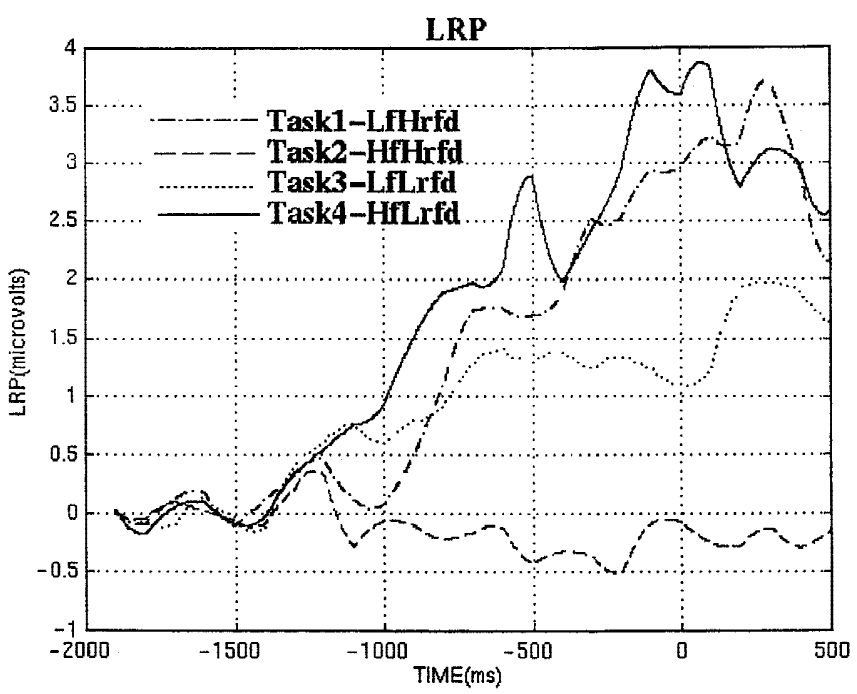

Figure 4. Lateralized readiness potentials from Study 2. Task 1 requires lower level of force and higher rate of force development. Task 2 requires higher level of force and higher rate of force development. Task 3 requires lower level of force and lower rate of force development. Task 4 requires higher level of force and lower rate of force development.

(LfLrfd). Negative lateralization $(\mathrm{LRP}=-0.477 \pm 0.227 \mu \mathrm{V})$ was observed when subjects performed Task 4 (HfLrfd), which required the lowest rate of force development $(14.4 \% \mathrm{MVF} / \mathrm{s})$, although higher nominal level of force (65\% MVF). The results of statistical analysis supported these observations. In particular, there was a significant effect for the rate of force development with the overall tendency toward larger LRP with higher rate of force development, $F(1,11)=5.92, p<.012$. The main effect of nominal force on LRP was not significant. Finally, there was a significant interaction between rate of force development and force, $F(1,11)=$ 5.68, $p<.022$. This interaction means that the movement tasks with higher nominal force that were performed with a higher rate of force development induced the largest LRPs.

Study 3. Grand-average waveforms of the force-onset synchronized LRP for the four experimental tasks are shown in Figure 5. As can be seen from this figure, the largest $\operatorname{LRP}(1.778 \pm 0.317 \mu \mathrm{V})$ was observed where subjects performed the repetitive task as fast as possible (RepSpd task). Less, but still significant lateralization $(1.379 \pm 0.193 \mu \mathrm{V})$ was observed for the discrete task under the speed condition (DisSpd task). Nonsignificant lateralization was observed when subjects performed the repetitive task under the accuracy conditions (RepAcc task) $(0.328 \pm 0.120 \mu \mathrm{V})$ and the discrete task under accuracy conditions (DisAcc task) $(-0.793 \pm$ $0.153 \mu \mathrm{V})$. In particular, the ANOVA revealed a significant effect for movement type with the overall tendency toward larger LRP for repetitive movement, $F(1,4)=4.43, p<.034$. A significant main effect of instruction was also found with larger LRP for speed, $F(1,4)=5.52, p<.013$. There was a significant interaction of movement type (discrete vs. repetitive) and instruction (speed vs. accuracy), $F(1,4)=5.23, p<.025$. Similar to Study 1 , post hoc analysis revealed that LRP values for RepSpd task were significantly larger than those for RepAcc task $(p<.05)$. LRP values for DisSpd task were significantly higher than those 

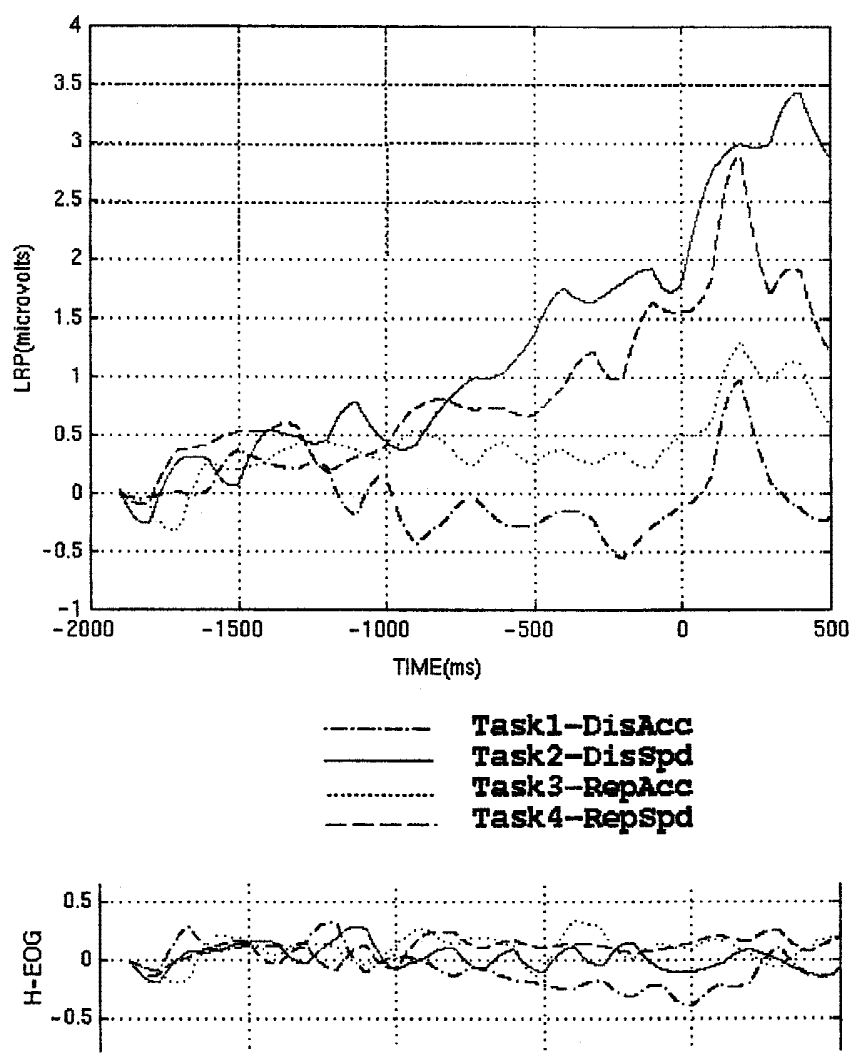

Figure 5. Lateralized readiness potentials (top) and horizontal eye movement (bottom) from Study 3. Task 1 is a discrete accuracy task; Task 2 is a discrete speed task; Task 3 is a repetitive accuracy task; Task 4 is a repetitive speed task.

for DisAcc task $(p<.01)$. In addition, LRP values for RepSpd task were significantly higher than those for DisSpd task $(p<$ $.05)$.

Study 4. Grand-average waveforms of the force-onset synchronized LRP for the four experimental tasks are shown in Figure 6. As can be seen from this figure, the largest LRP (2.712 \pm $0.498 \mu \mathrm{V}$ ) was observed during Task 2 (HfHrfd), which required highest nominal force $(65 \% \mathrm{MVF})$ and higher rate of force development $(31.8 \% \mathrm{MVF} / \mathrm{s})$. Slightly lower LRP values (2.474 \pm $0.342 \mu \mathrm{V}$ ) were observed when the same rate of force development $(31.8 \% \mathrm{MVF} / \mathrm{s})$ with less nominal force level (35\% MVF) was required (Task 1, LfHrfd). Even lower, but still significant LRP values $(1.285 \pm 0.347 \mu \mathrm{V})$ were observed when subjects performed Task 3 (LfLrfd). Negative lateralization (LRP = $-0.985 \pm 0.347 \mu \mathrm{V}$ ) was observed when subjects performed Task 4 (HfLrfd), which required the lowest rate of force development (14.4\% MVF/s), and a higher nominal level of force (65\% MVF). The results of statistical analysis supported these observations. In particular, the ANOVA revealed a significant effect for the rate of force development with the overall tendency toward larger LRP with higher rate of force development, $F(1,4)=9.08, p<.03$. The main effect of nominal force on LRP approached significance, $F(1,4)=2.62, p<.059$. Finally, there was a significant interaction of rate of force development and nominal force, $F(1,4)=4.45$, $p<.041$. This finding means that the movement tasks with a higher nominal force that were performed with a higher rate of force development induced the largest LRPs.
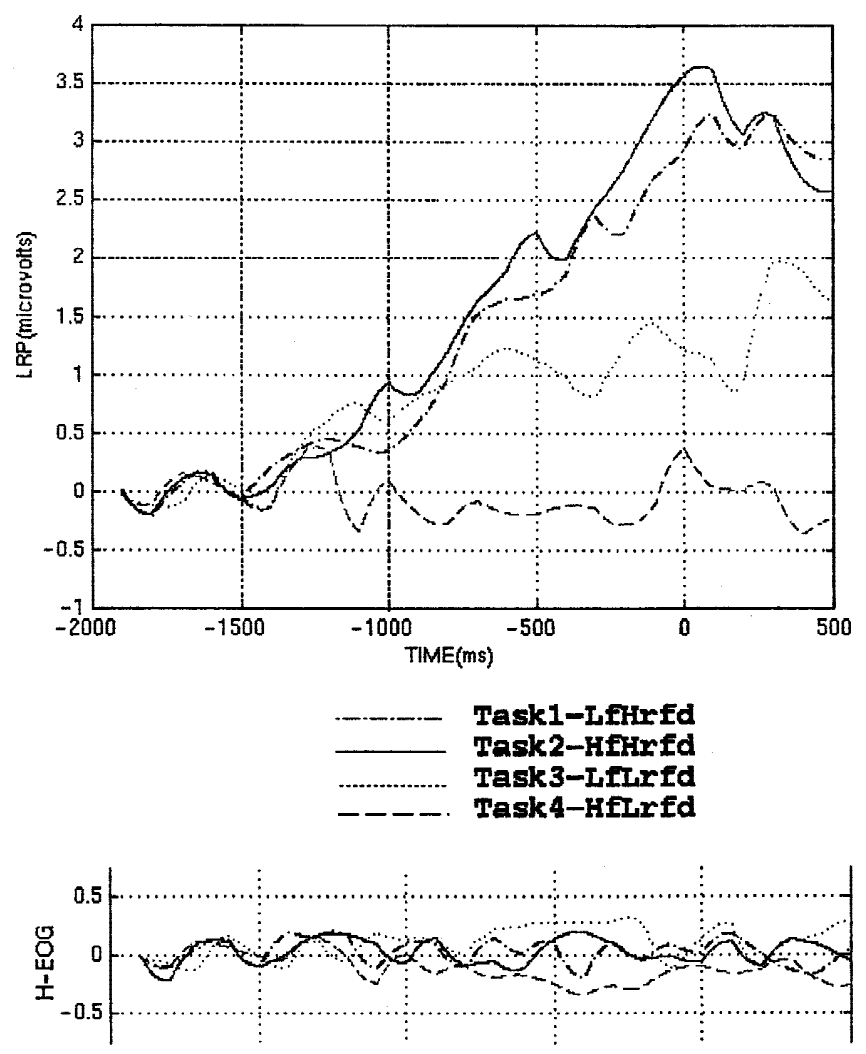

Figure 6. Lateralized readiness potentials (top) and horizontal eye movement (bottom) from Study 4. Task 1 requires lower level of force and higher rate of force development. Task 2 requires higher level of force and higher rate of force development. Task 3 requires lower level of force and lower rate of force development. Task 4 requires higher level of force and lower rate of force development.

\section{Discussion}

The major aim of our research was to examine the relationship between the force and rate of force development aspects of movement dynamics and the LRP. Consistent with the suggestion of Kutas and Donchin (1980), we used self-paced tasks to reflect more "pure motor preparation" MRPs. We began in Study 1 by investigating whether different speed and accuracy constraints in discrete and repetitive finger force production tasks influenced the LRP preceding these tasks. This study showed that speed tasks in contrast to accuracy tasks induced the greatest LRP regardless of whether the movement type was discrete or repetitive. These results were consistent with our previously published results reporting higher movement monitoring potentials for speed versus accuracy tasks. Further, in terms of movement type, repetitive tasks in comparison with discrete tasks induced the larger LRP in each of the speed and accuracy conditions. Given that speed rather than accuracy is associated with greater LRP changes, the question arose as to whether level of force or rate of force development was the major parameter. In Study 2, we found larger LRPs with the greatest rate of force development. This finding is also consistent with our previously published results showing that the amplitudes of MRP components preceding and accompanying finger force production movements were significantly correlated with rate of force development (Slobounov \& Ray, 1998; Slobounov et al., 1998). 
Taylor (1978) reported an increase in the BP potential as performance improved on the course of an experiment. This BP finding represents a potential confound in our Studies 1 and 2. Because subjects in Studies 1 and 2 performed all tasks with their right hand and then all tasks with their left, it is possible that lateralization differences or the lack thereof could have resulted from overall movement potential changes during the experiment. Further, because Studies 1 and 2 were initially performed for another purpose, horizontal eye movement was not recorded. This created another potential confound because the results could reflect eye movements toward the performing hand. To address these possibilities, our first two experiments were extended in Studies 3 and 4 by alternating the responding hand every fifth trial and by correcting for horizontal eye movement. The results of these replications (Figures 5 and 6) were identical with our first two experiments, thus ruling out the possibility that our findings represent movement potential changes across the experimental session. Further, Figures 5 and 6 help to rule out the alternative hypothesis that the results were related to eye movements toward the responding hand. Overall, our four studies demonstrate that preparation for differential rates of force development is a major component reflected in the LRP.

One finding of our LRP research is that with the larger rate of force development, level of force per se did not show as differentiated changes as with the lower rate of force development. This finding is consistent with that of Kutas and Donchin (1974, 1980), who reported that the amplitude of the MP increased with force output, but only at the higher level of force. One way to consider these results is to suggest a conditional preparatory process in which at higher levels of rate of force development level of force itself does not play an influence, whereas at the lower rate of force development level of force does play a role in the preparatory process.

Previous research addresses these results in three different ways. First, work by Coles (1989) reported that variability in human reaction time was related to variability in the LRP and this work has been supported by neuronal firing rate studies in the monkey cortex (cf. Requin, 1985). In our Studies 1 and 3, speeded tasks clearly influenced the LRP differently from accuracy tasks. In terms of accuracy tasks, Studies 1 and 3 showed that with accuracy instructions there are few indices of the tasks used that influenced the LRP. From this perspective, speed tasks are clearly handled differently from accuracy tasks by the motor system and may reflect a differential recruitment of motor units. Overall, we conclude that the LRP reflects a form of response activation that is sensitive to the movement parameters such as response speed but not to accuracy instructions. Second, Donchin, Gribova, Steinberg, Bergman, and Vaadia (1998) suggested that neuronal activity in the motor cortex is not reflective of contralateral representation in bimanual tasks. And third, findings in the area of timing control suggest that patients with cerebellar lesions are more accurate in finger tapping at a given frequency by the affected side when done in conjunction with tapping by the opposite side (other hand) (Helmuth \& Ivry, 1996). Taking these three lines of previous research together, one explanation for the lack of sensitivity of the LRP to tasks involving accuracy instructions would be that the control of pure accuracy tasks may involve contralateral and ipsilateral activation equally.

The findings from Studies 2 and 4 are inconsistent with the work of Sommer et al. (1994), who reported that both nominal force level and rate of force development did not influence the LRP. In our work, LRPs were indeed affected by rate of force development, regardless of the nominal force level of the planned movements. One characteristic of our work that differed from that of Sommer et al. was that our subjects viewed the target on a screen and had continuous feedback in terms of their response. The Sommer et al. study, on the other hand, gave subjects feedback only after they had completed a response. Thus, subjects in the two studies may have prepared differently for the movement task. This interpretation suggests that subjects who will not be receiving feedback will generate different preparatory motor commands from those expecting a feedback task. In a no-feedback condition, subjects may try to be correct at the very onset of the movement. If this were the case, then the no-feedback task becomes more of an accuracy task, which we found to produce less lateralization in Studies 1 and 3. For a feedback condition, on the other hand, subjects need only to generate necessary motor commands to start a movement, as they can correct the movement later (Magill, 1993). This method would reduce the accuracy requirement and allow for more speeded movements. This method is also supported by behavioral data in that our subjects were less accurate in tasks with higher rates of force production.

In addition to speed and accuracy interpretation of our results, there are other factors that may have played a role. As shown by Kutas and Donchin (1980), type of task (e.g., self-paced, signaled, warned, choice signaled, or choice warned) can influence preparatory MRPs. In a discussion of the supplementary motor area, Goldberg (1985) suggested that internally and externally guided movements are mediated by distinct motor areas. Likewise, neuronal discharges appear to be different in self-paced and triggered movements (Porter, 1985). Riehle and Requin (1989) further suggested two distinct types of motor preparation neurons that are involved in reaction time versus spontaneous movements. Although our work does not address this issue directly, such findings do form a basis for the differences found between traditional LRP studies using RT tasks and the present studies using self-paced tasks. Likewise, other factors may also be related to the presence of future feedback in our study and the lack of potential feedback in the Sommer et al. (1994) study. For example, subjects in the Sommer et al. study may have had the requirement of remembering the "feel" of a required response in terms of force and duration. The lack of continuous feedback may have required differential motor inhibitory processes (cf. De Jong, Coles, \& Logan, 1995) or added additional task requirements increasing complexity (cf. Hackley \& Miller, 1995), which have been shown to influence the LRP. Complexity or difficulty may have played a role in Studies 2 and 4, because the subjects were less accurate in tasks with higher slopes of force production and more error occurred at higher nominal force. Overall, our results are consistent with the proposition that the LRP reflects both preparation for responses and forthcoming movement complexity (Hackley \& Miller, 1995). Whether this type of interpretation calls into question the assumption that only motor factors influence the LRP is another question (cf. Eimer, 1998).

Our results were directed at preparations to respond rather than motor activity during the response itself. As such, the nature of the response tasks with continuous feedback clearly show motor preparations to be reflected in the LRP. In Studies 1 and 3, the results demonstrate that in a simple finger force production task, LRPs are more sensitive to speed than accuracy requirements. In a similar task, when the movement parameters are further differentiated factorially into level of force and rate of force development, LRPs are largest with a higher rate of force development. Thus, rate of force development appears to be an important parameter for determining amplitude of the LRP. 


\section{REFERENCES}

Carlton, L. G., Carlton, M. J., \& Newell, K. M. (1987). Reaction time and response dynamics. The Quarterly Journal of Experimental Psychology, 39A, 337-360.

Coles, M. G. H. (1989). Modern mind-brain reading: Psychophysiology, physiology and cognition. SPR Presidential address. Psychophysiology, $26,251-269$.

Crossman, E. R. F. W., \& Goodeve, R. J. (1983). Feedback control of hand-movement and Fitts' Law. Quarterly Journal of Experimental Psychology, 35A, 251-278.

De Jong, R., Coles, M., \& Logan, G. (1995). Strategies and mechanisms in nonselective and selective inhibitory motor control. Journal of Experimental Psychology: Human Perception \& Performance, 21, 498-511.

De Jong, R., Coles, M., Logan, G., \& Gratton, G. (1990). In search of the point of no return: The control of response processes. Journal of Experimental Psychology: Human Perception and Performance, 16, 164-182.

De Jong, R., Wierda, M., Mulder, G., \& Mulder, L. (1988). Use of partial stimulus information in response processing. Journal of Experimental Psychology: Human Perception and Performance, 14, 682-692.

Donchin, O., Gribova, A., Steinberg, O., Bergman, H., \& Vaadia, E. (1998). Primary motor cortex is involved in bimanual coordination. Nature, 395, 274-278.

Eimer, M. (1998). The lateralized readiness potential as an on-line measure of central response activation processes. Behavior Research Methods, Instruments and Computers, 30, 146-156.

Fitts, R. M. (1954). Information capacity of the human motor system in controlling the amplitude of movement. Journal of Experimental Psychology, 47, 381-391.

Goldberg, G. (1985). Supplementary motor area structure and function: Review and hypotheses. The Behavioral and Brain Sciences, 8, 567-616.

Gratton, G., Bosco, C., Kramer, A., Coles, M., Wickens, C., \& Donchin, E. (1990). Event-related brain potentials as indices of information extraction and response priming. Electroencephalography and Clinical Neurophysiology, 75, 419-432.

Hackley, S., \& Miller, J. (1995). Response complexity and precue interval effects on the lateralized readiness potential. Psychophysiology, 32, $230-241$.

Hancock, P., \& Newell, K. (1985). The movement speed-accuracy relationship in space time. In H. Aleur, U. Kleinbeck, \& K. H. Schmidt (Eds.). Motor behavior: Programming control and acquisition (pp. 153188). Berlin, Germany: Springer.

Helmuth, L. L., \& Ivry, R. B. (1996). When two hands are better than one: Reduced timing variability during bimanual movements. Journal of Experimental Psychology, 22, 278-293.

Jasper, H. H. (1958). The ten-twenty electrode system of the International Federation. EEG and Clinical Neurophysiology, 10, 371-375.

Kornhuber, H. H., \& Deecke, L. (1965). Hirnpotentialanderungen bei Willkurbewegungen und passiven Bewegungen des Menschen. Bereitschaftspotential und reafferente Potential. Pflügers Archiv für die Gesamte Physiologie des Menschen und der Tiere, 284, 1-17.

Kutas, M., \& Donchin, E. (1974). Studies of squeezing: Handedness, responding hand, response force, and asymmetry of readiness potential. Science, 186, 545-548.

Kutas, M., \& Donchin, E. (1980). Preparation to respond as manifested by movement related brain potentials. Brain Research, 202, 95-115.
Magill, R. A. (1993). Motor learning: Concepts and applications (pp. 100103). Madison, WI: Brown and Benchmark.

Meyer, D. E., Smith, J. E. K., Kornblum, S., Abrams, R. A., \& Wright, C. E. (1990). Speed-accuracy tradeoffs in aimed movements: Toward a theory of rapid voluntary action. In M. Jeannerod (Ed.), Attention and performance XIII (pp. 173-226). Hillsdale, NJ: Erlbaum.

Miller, J., Coles, M., \& Chakraborty, S. (1996). Dissociation between behavioral and psychophysiological measures of response preparation. Acta Psychologica, 94, 189-208.

Miller, J., \& Hackley, S. (1992). Electrophysiological evidence for temporal overlap among contingent mental processes. Journal of Experimental Psychology: General, 121, 195-209.

Newell, K. M., \& Carlton, L. G. (1985). On the relationship between peak force and peak force variability in isometric tasks. Journal of Motor Behavior, 17, 230-241.

Oldfield, R. C. (1971). The assessment and analysis of handedness: The Edinburgh inventory. Neuropsychologia, 9, 97-113.

Osman, A., \& Moore, C. (1993). The locus of dual-task interference: Psychological refractory effects on movement-related brain potentials. Journal of Experimental Psychology: Human Perception and Performance, 19, 1292-1312.

Osman, A., Moore, C., \& Ulrich, R. (1995). Bisecting RT with lateralized readiness potentials: Precue effects after LRP onset. Acta Psychologica, 90, 111-127.

Porter, R. (1985). Participation of SMA neurons in a "self paced" motor act. The Behavioral and Brain Sciences, 8, 596-597.

Requin, J. (1985). Looking forward to moving soon: Ante factum selective processes in motor control. In M. Posner \& O. Marin (Eds.), Attention and performance XI (pp. 147-167). Hillsdale, NJ: Erlbaum.

Riehle, A., \& Requin, J. (1989). Monkey primary motor and premotor cortex: Single-cell activity related to prior information about direction and extent of an intended movement. Journal of Neurophysiology, 61, 534-549.

Slobounov, S., \& Ray, W. (1998). Movement-related EEG potentials of isometric force production during speed and accuracy tasks. Experimental Brain Research, 123, 461-473.

Slobounov, S., Ray, W., \& Simon, R. (1998). Movement-related potentials accompanying unilateral finger movements with special reference to rate of force development. Psychophysiology, 35, 537-548.

Sommer, W., Leuthold, H., \& Ulrich, R. (1994). The lateralized readiness potential preceding brief isometric force pulses of different peak force and rate of force production. Psychophysiology, 31, 503-512.

Taylor, M. (1978). Bereitschaftspotential during the acquisition of a skilled motor task. Electroencephalography and Clinical Neurophysiology, 45, 568-576.

Ulrich, R., Leuthold, H., \& Sommer, W. (1998). Motor programming of response force and movement direction. Psychophysiology, 35, 721-728

Vaughan, H., Costa, L., \& Ritter, W. (1968). Topography of the human motor potential. Electroencephalography and Clinical Neurophysiology, 25, 1-10.

(Received October 5, 1998; ACCEPTED December 16, 1999) 